\title{
Tecnologías de la información y la comunicación (TICs) para la gamificación
}

Núria Pascual-Seva, María-Teresa Sebastiá-Frasquet, Chele Esteve Sendra, Sabina Asensio-Cuesta, María-Eugenia Babiloni Griñón, María-Teresa Palomares Figueres, Ana Portalés Mañanos y Maria Vargas Colás

Universitat Politècnica de València. Camino de Vera s/n. 46022 Valencia (España). nupasse@prv.upv.es

\begin{abstract}
Currently, the advances in information and communication technologies (ICTs) offer new tools to implement active teaching-learning methodologies. Gamification is a new teaching strategy, capable of implementing active methodologies in the classroom that can be facilitated by the use of ICTs. It allows the use of game mechanics in non-recreational environments to achieve educational objectives and it improves students' motivation. This has led to the introduction in the classroom of computer applications (or apps), computer programs and tools designed to allow undertaking various types of work.

The wide variety of apps available for gamification complicates their choice, not only for their topic, but also for their technology (mobile devices, tablets, and personal computers). Furthermore, although there are different apps which were not initially designed for their use in education, they can be used for this purpose. In response to this diversity, this article presents a review of different gamification experiences at higher education level. The main goal of this article is to provide a resource guide to the people interested in these apps.
\end{abstract}

Keywords: Gamification, Information and communications technology (ICTs), university teaching, active learning, apps

\section{Resumen}

Actualmente los avances en tecnologías la información y la comunicación (TICs) ofrecen nuevas herramientas para implementar metodologías de enseñanza-aprendizaje activas. Por otra parte la gamificación permite el empleo de mecánicas de juego en entornos no lúdicos para alcanzar 
Tecnologías de la información y la comunicación (TICs) para la gamificación

objetivos docentes y mejorar con ello la motivación de los alumnos. Se trata pues de una nueva estrategia docente, en auge en la docencia universitaria, capaz de implementar metodologías activas en el aula que puede ser facilitada por el uso de TICs. Esto ha dado lugar a la introducción en el aula de aplicaciones informáticas o apps (del inglés applications), programas informáticos diseñados como herramientas para permitir a un usuario realizar diversos tipos de trabajos.

La gran variedad de apps disponibles para la gamificación complica su elección, tanto como por su temática acomo por la tecnología utilizada (dispositivos móviles, tabletas y ordenadores personales). Además, hay distintas apps que aunque no fueran concebidas inicialmente para la gamificación pueden ser utilizadas para este fin. En respuesta a esta diversidad, el presente artículo realiza una revisión de las apps utilizadas en experiencias docentes de gamificación en el ámbito universitario. El objetivo es facilitar a los profesores interesados en desarrollar actividades de gamificación mediante TICs una guía de recursos.

Palabras clave: Gamificación, TICs, docencia universitaria, metodologías activas, apps

\section{Introducción}

La gamificación (gamification en el ámbito anglosajón) es el empleo de mecánicas de juego en entornos y aplicaciones no lúdicas con el fin de potenciar la motivación, la concentración, el esfuerzo y la fidelización (Deterding et al., 2011). Las actividades formativas pueden ser fácilmente transformadas en un juego, o gamificadas, gracias a que cumplen las siguientes premisas: (a) la actividad puede ser aprendida; (b) las acciones del usuario pueden ser medidas y (c) las retroalimentaciones pueden ser entregadas de forma oportuna al usuario (Gónzalez y Mora, 2015).

Para gamificar una actividad debe desarrollarse las dinámicas y las mecánicas. Las dinámicas son el concepto, la estructura implícita del juego, son las necesidades o motivaciones que tienen las personas: recompensa, competición, reconocimiento o estatus, autoexpresión, altruismo, superación, etc. Las mecánicas son los procesos que provocan el desarrollo del juego y pueden ser de distintos tipos: avatares, insignias, puntos, colecciones, rankings, niveles, bienes virtuales, misiones y retos, desafíos etc. En de Cea (2014) se puede encontrar una completa descripción de los distintos tipos de dinámicas y mecánicas. 
Tal y como afirma Valderrama (2015) para utilizar técnicas de gamificación, es necesario conocer las claves de la motivación para diseñar juegos que "enganchen”, a los distintos perfiles de estudiantes o jugadores: hedonistas vs luchadores, exploradores vs controladores, guerreros vs constructores, socializadores vs solitarios, caballeros andantes vs cazarecompensas.

Cuando empezamos a planificar una actividad de gamificación es importante tener en cuenta que implica algunos retos que deben ser resueltos según Herranz y Colomo-Palacios (2012): 1) No es fácil. Ni es fácil de gestionar, ni de determinar los objetivos, ni de medir y, además, implica cierta filosofía de prueba y error. 2) Existe el riesgo de caer en una pobre conceptualización y en una implementación poco acertada de los elementos del juego. Para evitar fallar es importante una buena planificación y selección de las dinámicas y mecánicas de juego así como de las herramientas para implementarlas. Así las experiencias llevadas ya al aula por otros compañeros nos pueden ayudar a gestionar mejor nuestra actividad de gamificación.

Con la finalidad de facilitar la selección de las herramientas más adecuadas para cada caso se fijan los objetivos del presente trabajo definidos en el siguiente apartado.

\section{Objetivos}

- Identificar aplicaciones informáticas potencialmente útiles para la gamificación en docencia universitaria.

- Elaborar una guía de aplicaciones TICs útiles para la gamificación.

- Establecer los beneficios del uso de TICs para la implementación de la gamificación.

\section{Desarrollo de la innovación}

El presente trabajo se desarrolla dentro del Proyecto de Mejora e Innovación Educativa (PIME) A05: Experiencia piloto de evaluación en distintas titulaciones de la UPV de la competencia transversal UPV "Responsabilidad ética, medioambiental y profesional", que se desarrollará durante los cursos académicos 2014/2015 y 2015/2016.

El objetivo de este PIME es diseñar mediante TICs una dinámica de juego, o gamificación, que permita adquirir y evaluar la citada competencia. Es importante destacar que no se pretender diseñar una nueva aplicación sino integrar distintos recursos existentes en una misma plataforma virtual. Así, el primer paso para poder diseñar la herramienta de gamificación es conocer los distintos recursos existentes y sus mecánicas y dinámicas de juego. Así, se podrá seleccionar aquellos que se adapten mejor a la finalidad de evaluar esta competencia transversal. Teniendo en cuenta un factor de gran importancia que es la variedad de perfiles de estudiantes que se dan en la Universitat Politècnica de València.

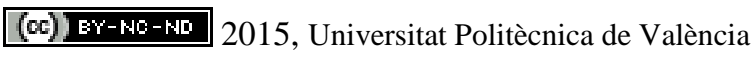

Congreso In-Red (2015) 
Se ha realizado una revisión bibliográfica a través de "Polibuscador" de la palabra clave "Gamificación”, tanto en español como en inglés (Gamification). Inicialmente no se filtró la búsqueda con términos adicionales como "docencia universitaria" (o "university teaching”). Aunque la primera aparición del concepto Gamificación data del 2002, no fue hasta la segunda mitad del 2010 cuando se extendió su uso (Herranz-Sánchez y ColomoPalacios, 2012). Dada la limitada franja de tiempo en la que se lleva utilizando la gamificación, se ha considerado interesante extender la búsqueda a distintos ámbitos educativos e incluso al ámbito de la empresa y el márquetin. Las estrategias de gamificación utilizadas en otros ámbitos pueden extrapolarse al aula en muchos casos y ayudan a plantear casos más prácticos.

\section{Resultados}

A partir de la revisión realizada se pueden estructurar los resultados en: 1) Catálogo de TICs para su uso en docencia universitaria, en el aula o fuera de ella, 2) Experiencias de uso de gamificación en el aula universitaria, y 3) beneficios observados del uso de técnicas de gamificación en docencia universitaria.

\subsection{Catálogo de TICs para su uso en docencia universitaria}

La revisión realizada ha permitido identificar técnicas de gamificación basadas en TICs. Además, se han identificado otras aplicaciones de software que suponen herramientas potenciales para la gamificación, tales como aplicaciones para el diseño de entornos virtuales, juegos de videoconsola, apps móviles, etc.

Las aplicaciones útiles para desarrollar estrategias de gamificación en el ámbito universitario pueden ser utilizadas en el aula, bien con el equipamiento propio del aula o en los distintos equipos personales de los alumnos como teléfonos móviles, tabletas u ordenadores portátiles. Además, estas aplicaciones se pueden articular a través de blogs que pueden servir de hilo conductor para la actividad de gamificación propuesta integrando distintas actividades y niveles de dificultad que permitan evaluar el nivel de consecución de la competencia que se esté trabajando.

En la siguiente tabla se presenta un catálogo de TICs útiles para su aplicación en actividades de gamificación docencia universitaria según la revisión realizada por Bruder (2014). 
Tabla 1. Catálogo de TICs. Fuente: Bruder (2014)

\begin{tabular}{cc} 
TICs & Fuente \\
\hline Level Up Tech Quest & http://leveluptechquest. wikispaces.com \\
Mashable & http://mashable.com \\
Team-Drill Head & (https://sites.google.com/site/teamdrillhead) \\
Sploder & www.sploder.com \\
Scratch & http://scratch.mit.edu \\
JeopardyLabs & www.jeopardylabs.com \\
Inform & http://inform7.com \\
PurposeGames & http://www.purposegames.com \\
\hline
\end{tabular}

A continuación se explican con mayor detalle otras de estas herramientas.

\subsubsection{Socrative}

Definición: Socrative (2015) es una herramienta para conocer la respuesta de los alumnos en tiempo real a través de ordenadores y dispositivos móviles. La aplicación se descarga de modo gratuito en el móvil o tableta. Permite trabajar desde el ordenador.

Comentarios: Permite la opción de utilizarse directamente en clase como repaso o encuesta. Se puede seleccionar la opción verdadera o falsa, respuestas cortas o de respuesta múltiple, o se puede hacer una pregunta en voz alta y los alumnos pueden contestarla. Es más participativo y se obtiene feedback de forma distinta y más real, sin recurrir a las preguntas tipo: ¿Me explico? ¿Os habéis enterado? ¿Seguro?, por parte del docente. Permite al profesor conocer de manera rápida y sencilla el nivel de conocimientos asimilados por parte de los alumnos, al realizar preguntas que son contestadas por los por ellos en tiempo real. Esta aplicación está siendo utilizada por algunos componentes de este grupo de innovación docente con resultados muy positivos.

Descarga: www.socrative.com (gratuito)

\subsubsection{QuizBean}

Definición: QuizBean (Bluehouse Gruop Production, 2015) permite realizar exámenes interactivos para un grupo específico de alumnos. Permite incrustar imágenes y todo tipo de contenido multimedia, compartir las pruebas en redes sociales, registrar las calificaciones obtenidas con la particularidad de ofrecer retroalimentación instantánea a los alumnos. Se parece a la app Socrative, ya que permite crear cuestionarios fomentando la

\section{(cc) EY-NC-ND 2015, Universitat Politècnica de València}


retroalimentación del alumno dependiendo de sus respuestas. Se crean quizzes o controles rápidos pero con opciones de calificaciones instantáneas, fomenta la retroalimentación, visualización móvil y la posibilidad de incrustar todo tipo de contenido multimedia.

Comentarios: Es muy visual para aquellas materias que precisen de imágenes y/o videos. En versión gratuita permite dar seguimiento hasta 51 alumnos y tiene un límite de 6 cuestionarios.

Descarga: www.quizbean.com

\subsubsection{Juegos didáctivos varios: Proyecto Descartes}

Definición: La herramienta de autor, Descartes (Proyecto Descartes, 2015), permite elaborar recursos didácticos interactivos que se embeben en páginas html. Esto permite interactuar con ellos en todos los dispositivos donde una página web tenga accesibilidad. Se trata de un applet (programa en lenguaje Java) configurable, diseñado para presentar interacciones educativas con números, funciones y gráficas. Descartes, puede ser utilizado por los autores de páginas Web educativas para enriquecer sus materiales con una amplia variedad de modelos matemáticos interactivos.

Comentarios: El Proyecto Prometeo, permite un nivel de dificultad superior. Configurar las herramientas es sencillo y puede aprenderse mirando las configuraciones de los ejemplos. Para crear una aplicación se recomienda comenzar con una aplicación que tenga cierta semejanza con la que se va a crear y modificarla paso a paso hasta conseguir el resultado que se busca.

Descarga: www.descartes.com

\subsubsection{Kuizza}

Definición: Kuizza (Kuizza, 2015) permite que los profesores creer un quiz o cuestionario y compartirlo con los alumnos vía Facebook, de manera pública. Esta aplicación permite revisar, solucionar y estudiar con las respuestas mediante tarjetas didácticas que se generan de forma establecida.

Comentarios: Es opción eficaz si se utiliza como material de estudio. Es de carácter público. No existen opciones privadas, las creaciones se hacen visibles para todo el mundo automáticamente. Así los estudiantes pueden revisarlo, solucionarlo y estudiar con las respuestas a través de las flashcards que se generan. Las flashcards o tarjetas de estudio que el servicio muestra con un solo clic, resultan muy atractivas.

Descarga: www.facebook.com/kuizza

\subsubsection{Aplicaciones de realidad virtual $3 D$}


Definición: Aplicaciones de realidad virtual 3D (v.g. Second Life, AR - Augmented Reality, 2015) El alumno podrá representarse en el mundo virtual y tomar decisiones recreando situaciones de un mundo real. Esto permitiría al profesor evaluar su nivel de adquisición de la competencia que desarrollamos en el citado PIME (Responsabilidad ética, medioambiental y profesional). Con la tecnología Realidad Aumentada (AR - Augmented Reality), la Realidad Virtual que está más extendida en la sociedad, se presenta ciertas características comunes como por ejemplo la inclusión de modelos virtuales gráficos 2D y 3D en el campo de visión del usuario. La diferencia principal versa en que la Realidad Aumentada no reemplaza el mundo real por uno virtual, sino al contrario, mantiene el mundo real que ve el usuario complementándolo con información virtual que se superpone al mundo real. El usuario jamás pierde el contacto con el mundo real. Esta experiencia le permite tener al alcance de su vista y al mismo tiempo puede interactuar con la información virtual superpuesta.

Comentarios: Las clases pueden hacer uso, entre otras cosas, de vídeo en tiempo real, presentaciones PowerPoint y chats. Conjuntamente, permite la interacción simultánea de varios agentes (como por ejemplo, la construcción colectiva de un objeto en Second Life), cosa imposible en los entornos clásicos de enseñanza online. Second Life es un juego masivo online que puede ser usado de modo gratuito y cuya apertura estructural lo hace atractivo como lugar virtual para la enseñanza. Otro ejemplo interesante es el proyecto Magic Book del grupo activo HIT de Nueva Zelanda. El alumno observa y lee en un libro real a través de un visualizador de mano y ve sobre las páginas reales los contenidos virtuales. El alumno de este modo ve una escena de realidad aumentada que reclame su atención y puede introducirse dentro de la escena y experimentar en un entorno virtual inmersivo.

Descarga: www.secondlife.com

\subsubsection{Videojuegos: Minecraft}

Definición: Minecraft (Romero, 2013) este videojuego tiene una versión educativa denominada MinecraftEDU que se usa en más de 1000 colegios entre Nueva York y Finlandia. Esta versión educativa permite crear "zonas de trabajo" para preparar las clases y otras herramientas, junto con la posibilidad de mover al alumno en el mapa o mandar mensajes.

Descarga: www.minecraftedu.com

\subsection{Experiencias de gamificación en docencia universitaria}


Tecnologías de la información y la comunicación (TICs) para la gamificación

En la siguiente tabla se resume la información analizada sobre la implementación de estrategias de gamificación facilitadas por TICs en docencia principalmente universitaria. 


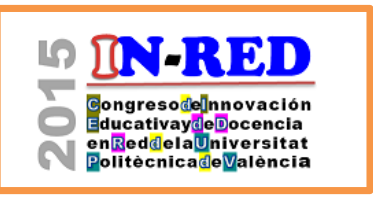

Congreso In-Red 2015 Universitat Politècnica de València Doi: http://dx.doi.org/10.4995/INRED2015.2015.1594

Tabla 1. Experiencias de Gamificación en docencia mediadas por TICs

\begin{tabular}{|c|c|c|c|c|}
\hline Asignatura & Titulación & Soporte para la Gamificación & Componentes & Referencia \\
\hline Educación Física & E.S.O. & Play the Game, Google site & $\begin{array}{l}\text { Insignias, puntos, recompensas, } \\
\text { retos, tablas de clasificación }\end{array}$ & Monguillot et al. 2015 \\
\hline $\begin{array}{l}\text { Gestión de } \\
\text { empresas mediante } \\
\text { simulaciones }\end{array}$ & $\begin{array}{l}\text { Universitaria (no } \\
\text { especificada) }\end{array}$ & Juegos de simulación & Sin información & Urquidi y Calabor 2014 \\
\hline No especificada & $\begin{array}{l}\text { Audiovisual, Sistemas de } \\
\text { Telecomunicación, Mecánica, } \\
\text { Organización Industrial }\end{array}$ & $\begin{array}{l}\text { Interwrite response, Quiz } \\
\text { show (cuestiones) }\end{array}$ & $\begin{array}{c}\text { Niveles, tablas de clasificación, } \\
\text { premios }\end{array}$ & Castilla et al. 2013 \\
\hline $\begin{array}{c}\text { Sistemas } \\
\text { de Interacción } \\
\text { Persona- } \\
\text { Computador }\end{array}$ & Ingeniería Informática & $\begin{array}{l}\text { Google drive, } \\
\text { Google }+\end{array}$ & $\begin{array}{l}\text { Niveles, puntos, } \\
\text { recompensas }\end{array}$ & $\begin{array}{l}\text { González y } \\
\text { Mora } 2015\end{array}$ \\
\hline Lenguas extranjeras & $\begin{array}{c}\text { Programa de Lenguas } \\
\text { Modernas }\end{array}$ & $\begin{array}{l}\text { Babel } \\
\text { (videojuego) }\end{array}$ & & $\begin{array}{l}\text { Hernández } \\
\text { et al. }\end{array}$ \\
\hline Lengua Inglesa III & Diplomatura en Turismo & Qstream & Puntos, & Ibañez 2015 \\
\hline
\end{tabular}

(c) EY-NC-ND 2015, Universitat Politècnica de València

Congreso In-Red (2015) 
Tecnologías de la información y la comunicación (TICs) para la gamificación

\begin{tabular}{|c|c|c|c|c|}
\hline Asignatura & Titulación & Soporte para la Gamificación & Componentes & Referencia \\
\hline $\begin{array}{l}\text { para el Turismo } \\
\text { Inglés II para } \\
\text { Turismo }\end{array}$ & Grado en Turismo & (cuestionarios) & $\begin{array}{l}\text { clasificaciones, } \\
\text { progresión }\end{array}$ & \\
\hline Alemán nivel A1 & Univsersitario Sin especificar & $\begin{array}{l}\text { Opensim (Mundos } \\
\text { virtuales 3D) }\end{array}$ & Puntos & $\begin{array}{l}\text { Palomo et } \\
\text { al. } 2014\end{array}$ \\
\hline $\begin{array}{c}\text { Español como } \\
\text { Lengua Extranjera }\end{array}$ & $\begin{array}{c}\text { Centro de enseñanza de } \\
\text { español como lengua } \\
\text { extranjera }\end{array}$ & $\begin{array}{l}\text { Videojuegos, } \\
\text { juegos de rol }\end{array}$ & & Riquel 2014 \\
\hline $\begin{array}{l}\text { Dirección } \\
\text { Estratégica y } \\
\text { Dirección de la } \\
\text { Producción }\end{array}$ & Universitario Sin especificar & $\begin{array}{l}\text { Dilema del } \\
\text { Estudiante } \\
\text { (simulaciones) }\end{array}$ & Puntos, rondas & $\begin{array}{l}\text { Graeml et al. } \\
2013\end{array}$ \\
\hline
\end{tabular}

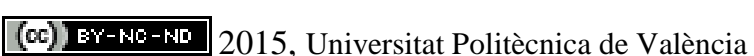




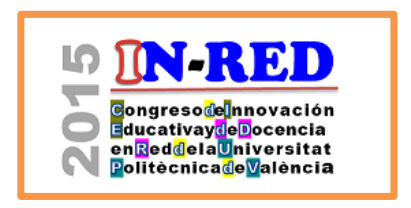

\subsection{Análisis de la gamificación en docencia universitaria}

A partir del estudio de las experiencias en gamificación resumidas en el anterior epígrafe podemos decir que la gamificación en el aula mediante el apoyo de TICs es bastante reciente, y casi todas las experiencias datan a partir de 2013.

Esto conlleva a que los resultados publicados sobre los beneficios/inconvenientes de utilizar esta técnica se relacione con estudios puntuales realizados en un único curso académico y con grupos de alumnos en muchas ocasiones también reducidos.

Así por ejemplo Urquidi y Calabor (2014) publican los resultados observados en el año académico 2011-2012 en un curso de 40 horas. Ibañez (2015) trabajo con un grupo de 27 voluntarios, aunque solamente completaron el proyecto 16 estudiantes. Castilla et al. (2013) en una muestra total de 44 estudiantes comprueba que en los casos en que los estudiantes fueron evaluados con técnicas de gamificación lograron mejores resultados, respondiendo a un número total de preguntas mucho mayor que los estudiantes evaluados con un test tradicional.

Monguillot et al. (2015) trabaja con un grupo de 102 alumnos en el curso 2013/14 y concluye que el $77 \%$ de alumnado valora la gamificación cómo una estrategia de motivación. A su vez destaca que los retos más atractivos han sido los que implican la interacción con otros compañeros de forma cooperativa. Todos los estudios analizados coinciden en la gran ventaja que suponen las dinámicas de gamificación para el trabajo en grupo. Por ejemplo, Gonzalez y Mora (2015) destacan también el importante componente social, es decir, el contar con otras personas con las que competir, colaborar y comparar logros. También Riquel (2014) coincide en esta observación. En este juego social los objetivos pueden ser competitivos o colaborativos, es importante conocer previamente el perfil de los estudiantes y escoger la dinámica de juego que más se adapte a sus características. En este sentido personalizar los retos permite además atender a diferentes ritmos de aprendizaje (Monguillot et al., 2015).

Otra gran ventaja de la gamificación es la posibilidad de motivar a los estudiantes para aplicar lo aprendido en sus proyectos de futuro, laborales y personales. González y Mora (2015) trabajaron con un grupo de 100 estudiantes de tercer curso del Grado en Ingeniería Informática, el 71,7\% de ellos tenía expectativas con los contenidos de la asignatura para aplicarlos a sus proyectos futuros.

Coincidimos con Romero y Rojas (2013) en la potencialidad de implementar técnicas de gamificación dentro del blended learning. Agregar este componente es un buen modo de

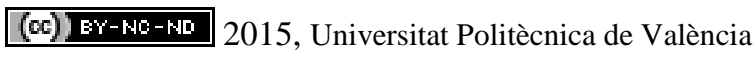

Congreso In-Red (2015) 
mantener el interés de los alumnos, que puede traer consigo beneficios al proceso de enseñanza-aprendizaje a través de un elemento diferenciador y creativo.

Cabe destacar que entre los estudios analizados predomina la gamificación mediante juegos de cuestiones (Castilla et al., 2013; Ibañez, 2015). En principio podemos pensar que estas actividades requieren un tiempo de preparación menor que otras que utilizan videojuegos y juegos de simulación, aunque no existe ningún dato al respecto del tiempo invertido en el diseño de las estrategias de gamificación. Riquel (2014) destaca en sus conclusiones como "el docente debe pensar muy bien el objetivo que pretende conseguir mediante los videojuegos o la actividad creada con las estrategias de los mismos, así como sopesar el esfuerzo de preparación en relación al impacto deseado en el aprendizaje". Lo que sí es cierto es que la utilización de TICs para implementar las estrategias de gamificación permite una gestión del tiempo más eficaz que su no utilización, además de otras ventajas añadidas como el atractivo visual, más facilidad de feedback, etc. Esta utilidad ha sido reconocida por otros autores como Cantador (2012) quien desarrolla una brillante actividad de gamificación en Informática aplicada en primero de Grado en Ingeniería Química con Aprendizaje Basado en Problemas sin apoyo de TICs. En las conclusiones de su trabajo Cantador (2012) reconoce que el uso de TICs podría facilitar en gran medida esta actividad.

La TIC ideal a utilizar en cada caso debe elegirse en función del objetivo de aprendizaje perseguido, teniendo en cuenta el perfil de los alumnos para escoger las dinámicas y mecánicas de juego que resulten más motivadoras. Asimismo, es importante que el profesor se sienta cómodo con la herramienta y es recomendable en las primeras experiencias optar por utilizar las más simples que puedan cumplir con el objetivo, para ir mejorando con la experiencia.

En todos los trabajos analizados se destacan las bondades de la gamificación en la docencia, pero es necesaria una continuidad en el uso de estas técnicas para poder acreditar los resultados de forma fehaciente.

\section{Conclusiones}

La gamificación es una técnica de aprendizaje que ha sido aplicada en la docencia no universitaria. En la actualidad y en el marco del espacio europeo de aprendizaje dicha técnica está siendo cada vez más aplicada en el ámbito universitario dado que permite la implementación de metodología activas.

Existe una gran variedad de aplicaciones de software disponibles para la aplicación de la gamificación en la docencia universitaria, tanto en el aula como en la formación a distancia. El problema que se plantea es decidir cuál aplicar, cómo y en qué contexto. El presente 
trabajo trata de dar apoyo al docente a la hora de responder a dicha cuestión. Para ello se presenta una recopilación de aplicaciones de software y posibles usos para la gamificación.

La mayor trascendencia de este trabajo es la elaboración de una guía que recopila diferentes aplicaciones de software y propone actividades de gamificación en las que pueden ser utilizadas.

\section{Referencias}

\section{Revistas}

BRUDER, P. (2014). Game on: gamification in the classroom. New Jersey Education Association. $<$ http://www.njea.org/news-and-publications/njea-review/may-2014/gamification-in-the-classroom $>$ [Consulta: 30 de mayo de 2015].

DETERDING, S., DIXON D., KHALED, R. y NACKE, L.(2011). Gamification: Toward a Definition. ACM 978-1-4503- 0268-5/11/05

GONZALEZ GONZALEZ, C.S., MORA CARREÑO, A. (2015). Técnicas de gamificación aplicadas en la docencia de Ingeniería Informática. Revista Hispanoamericana de Educación Universitaria de la InformáticaReVisión; $\quad$ Vol $8, \quad$ No $1 . \quad$ Editorial $\quad$ CSIC. $<$ http://www.aenui.net/ojs/index.php?journal=revision\&page=article\&op=view\&path\%5B\%5D=152 $>$ [Consulta: 30 de mayo de 2015].

HERNANDEZ MORALES, O.C., ARCINIEGAS OVALLE, F., GARCIA MONSALVE, L.S. y RODRIGUEZ SANCHEZ, C.A. Babbel: una experiencia de gamificación en el campo de las lenguas extranjeras

$<$ http://www.virtualeduca.pro/documentos/23/HERNANDEZ,\%20ARCINIEGAS,\%20GARCIA,\%20 MONSALVE.pdf>. [Consulta: 30 de mayo de 2015].

HERRANZ SANCHEZ, E. y COLOMO-PALACIOS, R. (2012). La Gamificación como agente de cambio en la Ingeniería del Software. España: AEMES Revista de Procesos y Métricas, Vol. 9 (2) (2012) <http://www.aemes.org/index.php/revista-de-procesos-y-metricas/numeros-publicados/ano2012-volumen-9-numero-2/articulos/405-la-gamificacion-como-agente-de-cambio-en-la-ingenieriadel-software > [Consulta: 30 de mayo de 2015].

IBAÑEZ MORENO, A. (2015). La gamificación para (auto)evaluar las competencias léxicogramaticales en el aula de inglés como segunda lengua en el contexto de la enseñanza a distancia: un estudio de caso. Verbeia Número 0. Universidad Camilo José Cela. $<$ http://www.ucjc.edu/wp-content/uploads/6.Ana-Ibanez-Moreno.pdf > [Consulta: 30 de mayo de 2015].

MONGUILLOT HERNANDO, M. GONZALEZ AREVALO, C., ZURITA MON, C., ALMIRALL BATET, L. Y GUITERT CATASUS, M. (2015) Play the Game: gamificación y hábitos saludables en educación física. Apunts. Educación Física y Deportes n. $^{\circ}$ 119, pp. 71-79. 
Tecnologías de la información y la comunicación (TICs) para la gamificación

http://dx.doi.org/10.5672/apunts.2014-0983.es.\%282015/1\%29.119.04 [Consulta: 30 de mayo de 2015].

PALOMO DUARTE, M. BERNS, A. Y GOMEZ SANCHEZ, R. (2104) Análisis de interacción en mundos virtuales con gamificación: una experiencia en alemán como lengua extranjera. Proyectos de INNOVACIÓN Y MEJORA DOCENTE PI1_14_063, Julio de 2014.

ROMERO SANDÍ, H. y ROJAS RAMÍREZ, E. (2013). La Gamificación como participante en el desarrollo del B-learning: Su percepción en la Universidad Nacional, Sede Regional Brunca. Eleventh LACCEI Latin American and Caribbean Conference for Engineering and Technology (LACCEI'2013) "Innovation in Engineering, Technology and Education for Competitiveness and Prosperity” August 14 - 16, 2013 Cancun, Mexico.

URQUIDI MARTIN, A.C. Y CALABOR PRIETO, M.S. (2014) Aprendizaje a través de juegos de simulación: un estudio de los factores que determinan su eficacia pedagógica. EDUTEC. Revista Electrónica de Tecnología Educativar ISSN 1135-9250 Núm. 47 <http://www.edutec.es/revista/index.php/edutec-e/article/view/75> [Consulta: 30 de mayo de 2015].

VALDERRAMA, (2015) Los secretos de la gamificación: 10 motivos para jugar. Capital Humano Núm. 295, 72-78. <http://www.capitalhumano.es> [Consulta: 30 de mayo de 2015].

\section{Ponencias de congresos}

CASTILLA, G., ROMANA, M. G., LOPEZ-TERRADAS, B. (2013) «Concursando en el aula: la gamificación mediante quiz-show como herramienta de dinamización docente » En : X Jornadas Internacionales de Innovación Universitaria. Educar para transformar. Universidad Europea. Disponible

<http://abacus.universidadeuropea.es/bitstream/handle/11268/2043/1572.pdf?sequence=1 $>$ [Consulta: 30 de mayo de 2015].

FONSECA DIAZ, A., FRIAS LOPEZ, D. (2014). La gamificación aplicada a la mentoría mediante dinámicas de team building para el fortalecimiento de equipos de trabajo. XI Jornadas Internacionales de Innovación Universitaria Educar para transformar. Universidad Europea. Disponible en : $<$ http://hdl.handle.net/11268/3568> [Consulta: 30 de mayo de 2015].

GRAEML, F.R., GRAEML, A.R., PlATERO, M. y MASSOTE, A. (2013) "Colaborar o no colaborar: el dilema del estudiante”. López, D. (coord.) En: X Jornadas Internacionales de Innovación Universitaria. Educar para transformar y IX Jornadas Internacionales de Innovación Universitaria Retos y oportunidades del desarrollo de los nuevos títulos en educación superior. Universidad Europea. Disponible en <http://hdl.handle.net/11268/2021> [Consulta: 30 de mayo de 2015].

\section{Tesis, trabajo final, tesina}

CANTADOR, I.. (2012) Competition as a Teaching Methodology: An Experience Applying Problembased Learning and Cooperative Learning. Directora: Rora M. Carro. Tesis presentada para la obtención del título de Experto en Docencia Universitaria, en la UNIVERSIDAD AUTONOMA DE 
MADRID (UAM). Madrid, España,

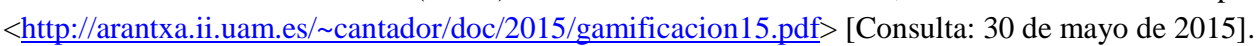

DE CEA BELLO, A. (2014). Diseño y desarrollo de aplicaciones software para la creación de actividades docentes con elementos de Gamificación. Trabajo Final de Grado. Madrid: UNIVERSIDAD AUTONOMA DE MADRID, <https://repositorio.uam.es/handle/10486/661000 > [Consulta: 30 de mayo 2015]

RIQUEL GARCÍA, A.M. (2014). Incrementando la motivación en la clase de ELE: el uso de la gamificación en el aula Trabajo Final de Máster. UNIVERSIDAD DE SEVILLA <https://idus.us.es/xmlui/bitstream/handle/11441/23261/TFM\%20Riquel\%20Garc\%C3\%ADa.pdf ?sequence=1\&isAllowed $=\mathrm{y}>$ [Consulta: 30 de mayo de 2015].

\section{Simulaciones y juegos (software)}

BLUEHOUSE GROUP PRODUCTION. 2015. <https://www.quizbean.com/home> [Consulta : 9 de abril de 2015]

KUIZZA, 2015. < http://www.kuizza.com> [Consulta : 9 de abril de 2015]

MINECRAFTEDU , 2015. <http://minecraftedu.com/> [Consulta : 9 de abril de 2015]

Proyecto Descartes, 2015. <http://newton.proyectodescartes.org/juegosdidacticos> [Consulta : 9 de abril de 2015]

SECOND LIFE, 2015. > http://www.secondlife.com> [Consulta : 9 de abril de 2015]

SOCRATIVE, 2015. <http://www.socrative.com> [Consulta : 9 de abril de 2015] 